\title{
Separability conditions from entropic uncertainty relations
}

\author{
Vittorio Giovannett:* \\ Research Laboratory of Electronics, MIT - Cambridge, MA 02139, USA
}

\begin{abstract}
We derive a collection of separability conditions for bipartite systems of dimension $d \times d$ which is based on the entropic version of the uncertainty relations. A detailed analysis of the two-qubit case is given by comparing the new separability conditions with existing criteria.
\end{abstract}

PACS numbers: 03.65.Ud, 03.65.Ta, 03.67.-a

Separability is the property that distinguishes statistical ensembles that have a classical description from the ones that need a quantum description. As a matter of fact, the definition of entanglement is the formal negation of the separability condition [1]. In spite of this clear logical distinction between separable and entangled states, the borderline between these two sets is very difficult to characterize in practice. In the case of bipartite systems, many necessary conditions for separability (i.e. criteria for entanglement) have been proposed 2 $, 3,4,5,6,7,8,9,10]$. Some of these conditions pertain to geometrical properties of separable states which are difficult to observe experimentally 2]. The ones that have a better chance to become operative procedures for testing the presence of entanglement are those criteria that establish boundaries either on the expectation values 3,4$]$, or on the statistical variances [5, 6, 7, 8, 9, 10] of some observables. These last separability conditions take advantage of the fact that, when specified on nonentangled states, the uncertainty relations of a collection of nonlocal observables $\hat{X}_{1}, \hat{X}_{2}, \ldots$ (i.e., observables that act nontrivially in both the Hilbert spaces which constitute the bipartite system) are forced to satisfy lower bounds, which are higher than the ones they have to obey when applied to generic states [5, 6, 7, 8, 9, 10]. The reason for this is that, in general, $\hat{X}_{1}, \hat{X}_{2}, \ldots$ possess only entangled eigenstates: on such states these observables are allowed to achieve the minimum values of their uncertainties. On the other hand, because no common eigenstate of $\hat{X}_{1}, \hat{X}_{2}, \ldots$ is separable, it is not possible to minimize the uncertainties of all these observables simultaneously on such configurations.

In this paper we propose an approach to the separability problem of a bipartite system based on the entropic uncertainty relations in place of the usual Heisenberg-like uncertainty relations. A somehow analogous endeavor has been undertaken in [11] where the standard Bell inequalities were written in terms of entropic quantities. The strategy we propose takes direct advantage of the geometrical structure of the tensor product Hilbert space of the system and underlines the connections between uncertainty relations and entanglement [12]. The material

\footnotetext{
*Recently at: NEST-INFM \& Scuola Normale Superiore, Piazza
} dei Cavalieri 7, I-56126, Pisa, Italy. is organized as follows. In Sec. I] we give a brief review of the entropic relations. In Sec. III by analyzing the simple case of a two-qubit system, we show how it is possible to generate a new class of separability conditions using entropic uncertainty relations of nonlocal operators. In Sec. III] we generalize these results to bipartite systems of dimension $d \times d$ with $d \geqslant 2$.

\section{ENTROPIC UNCERTAINTY RELATIONS}

Entropic uncertainty relations have been proposed as an alternative to the standard Heisenberg-like relations, in the case of observables with nontrivial C-number commutators 12, 13, 14, 15, 16, 17]. The basic idea of this approach is to replace the statistical variance with the Shannon entropy as an estimator of the uncertainties associated with the measurement process. Consider for instance an observable $\hat{X}$ with $K$ distinct eigenvalues $x_{1}, \ldots x_{K}$ and spectral decomposition

$$
\hat{X}=\sum_{k=1}^{K} x_{k} \mathbb{X}_{k}
$$

with $\mathbb{X}_{k}$ the projector in the eigenspace of $\hat{X}$ relative to the eigenvalue $x_{k}$. Given a state $\rho$ of the system, we define the entropic uncertainty of $\hat{X}$ as

$$
H(\mathbb{X}, \rho) \equiv-\sum_{k=1}^{K} P_{k} \ln P_{k}
$$

where $P_{k} \equiv \operatorname{Tr}\left(\mathbb{X}_{k} \rho\right)$ is the probability of finding the state $\rho$ in the $k$ th eigenspace. In Eq. (2) the symbol $H(\mathbb{X}, \rho)$ is used to underline the dependence of this quantity from the projectors $\mathbb{X}_{k}$ defined in Eq. (1): this notation allows us to distinguish the definition of entropic uncertainty of $\hat{X}$ introduced here from the standard definition $H(\hat{X}, \rho)$ adopted elsewhere [18]. $H(\mathbb{X}, \rho)$ can be used to estimate the uncertainty of the outcome of a measurement of $\hat{X}$ on the state $\rho$. In fact, if $\rho$ is one of the eigenvectors belonging, say, to the $k_{0}$ th eigenspace, then $P_{k}=\delta_{k, k_{0}}$ and $H(\mathbb{X}, \rho)$ nullifies. On the contrary, if $\rho$ is an equally weighted superposition or mixture of all the eigenstates of $\hat{X}$, the measurement result is maximally undetermined and $H(\mathbb{X}, \rho)$ achieves its maximum value $\ln K$. Now introduce a second observable $\hat{Y}$ with 
eigenspaces projectors $\left\{\mathbb{Y}_{k^{\prime}}\right\}$. Following the derivation of 17], it is straightforward to show that an entropic uncertainty relation applies, i.e.

$$
H(\mathbb{X}, \rho)+H(\mathbb{Y}, \rho) \geqslant-2 \ln \left(\max _{k, k^{\prime}}\left\|\mathbb{X}_{k} \mathbb{Y}_{k^{\prime}}\right\|\right),
$$

where $H(\mathbb{Y}, \rho)$ is the entropic uncertainty of $\hat{Y}$ and where $\|\mathcal{O}\| \equiv \max _{|\psi\rangle} \| \mathcal{O}|\psi\rangle \|$ is the norm of the operator $\mathcal{O}$. In our approach Eq. (3) replaces the standard uncertainty relation which involves the product of the statistical variances of the two operators. These two relations are not completely equivalent, but both predict that when $\hat{X}$ and $\hat{Y}$ commute, no constraint is imposed on the accuracy with which we can measure them on the same state (see for example [14, 15]).

\section{SEPARABILITY CONDITIONS FOR QUBITS}

A separable state of a bipartite system $\mathcal{S}$ composed of subsystems $\mathcal{A}$ and $\mathcal{B}$ is any density matrix $\rho_{\text {sep }}$ that can be expressed as a convex combination of tensor product states, as

$$
\rho_{\text {sep }}=\sum_{n} \lambda_{n}\left|\psi_{n}\right\rangle_{a}\left\langle\psi_{n}|\otimes| \phi_{n}\right\rangle_{b}\left\langle\phi_{n}\right|,
$$

with $\left|\psi_{n}\right\rangle_{a}$ and $\left|\phi_{n}\right\rangle_{b}$ pure states of the subsystem $\mathcal{A}$ and $\mathcal{B}$ respectively, and $\lambda_{n} \geqslant 0, \sum_{n} \lambda_{n}=1$. Any state of this form is, by definition, not entangled [1]. The aim of this paper is to give a class of entropic relations, such as Eq. (3), that can be violated by entangled states but not by the states $\rho_{\text {sep }}$. We begin by considering the simple case where $\mathcal{A}$ and $\mathcal{B}$ are both qubits: the method will be then extended to subsystems of higher dimension in Sec. III]

\section{A. First example}

Consider the following observables

$$
\hat{X} \equiv \sigma_{a}^{(1)} \otimes \sigma_{b}^{(1)}, \quad \hat{Y} \equiv \sigma_{a}^{(2)} \otimes \sigma_{b}^{(2)},
$$

where $\sigma_{s}^{(j)}$, for $j=1,2,3$ and $s=a, b$, are the Pauli operators acting on the $s$ qubit. Because $\hat{X}$ and $\hat{Y}$ commute, the right-hand-side of Eq. (3) vanishes and no lower bound is required to the sum of the entropic uncertainties of these operators. For example, one can nullify both $H(\mathbb{X}, \rho)$ and $H(\mathbb{Y}, \rho)$ by choosing $\rho$ to be one of the four maximally entangled elements of the Bell basis

$$
\begin{aligned}
& \left|\Psi_{1}\right\rangle=(|00\rangle+|11\rangle) / \sqrt{2} \\
& \left|\Psi_{2}\right\rangle=(|00\rangle-|11\rangle) / \sqrt{2} \\
& \left|\Psi_{3}\right\rangle=(|01\rangle+|10\rangle) / \sqrt{2} \\
& \left|\Psi_{4}\right\rangle=(|01\rangle-|10\rangle) / \sqrt{2},
\end{aligned}
$$

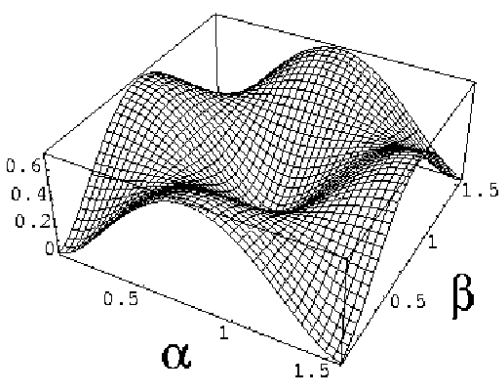

FIG. 1: Entropic uncertainty relations for the pure separable state $\left|\Psi_{\text {sep }}\right\rangle$ of Eq. (8). The plot shows the difference between the right-hand-side of Eq. (12) evaluated for $\delta=-\gamma=\pi / 2$ and the lower bound $\ln 2$ : this function is never negative.

where, for instance, $|01\rangle$ is the state $|0\rangle_{a} \otimes|1\rangle_{b}$ with $|0\rangle$ and $|1\rangle$ being the eigenvectors of the the Pauli operator $\sigma^{(1)}$ relative to the eigenvalues +1 and -1 , respectively. On the other hand, if we consider separable states $\rho_{\text {sep }}$, it is possible to show that the following inequality applies,

$$
H\left(\mathbb{X}, \rho_{\text {sep }}\right)+H\left(\mathbb{Y}, \rho_{\text {sep }}\right) \geqslant \ln 2 .
$$

This relation can be used to test the presence of entanglement in the system: if some state violates it, such a state cannot be separable. Since the Shannon entropy is a concave function [19], to prove Eq. (7) it is sufficient to show that it applies to any pure separable state [14, 15, 17]

$$
\left|\Psi_{s e p}\right\rangle=|\psi\rangle_{a} \otimes|\phi\rangle_{b},
$$

with

$$
\begin{aligned}
|\psi\rangle_{a} & =\cos \alpha|0\rangle_{a}+e^{i \delta} \sin \alpha|1\rangle_{a} \\
|\phi\rangle_{b} & =\cos \beta|0\rangle_{b}+e^{i \gamma} \sin \beta|1\rangle_{b},
\end{aligned}
$$

where $\alpha, \beta, \delta$ and $\gamma$ are real parameters. The observable $\hat{X}$ has the eigenvalues +1 and -1 , which are both two-time degenerate and have eigenspaces generated by the vectors $\{|00\rangle,|11\rangle\}$ and $\{|01\rangle,|10\rangle\}$, respectively. The probabilities of finding the state $\left|\Psi_{\text {sep }}\right\rangle$ in these eigenspaces can be then expressed as,

$$
\begin{aligned}
& P_{+}=|\cos \alpha \cos \beta|^{2}+|\sin \alpha \sin \beta|^{2} \\
& P_{-}=1-P_{+} .
\end{aligned}
$$

Consequently the entropic uncertainty of $\hat{X}$ is $H\left(\mathbb{X},\left|\Psi_{\text {sep }}\right\rangle\right)=\mathcal{H}_{2}\left(P_{+}\right)$with

$$
\mathcal{H}_{2}(x) \equiv-x \ln x-(1-x) \ln (1-x),
$$

the binary entropy function. In the same way we can calculate the entropic uncertainty of the operator $\hat{Y}$ and show that the following relation applies,

$$
\begin{gathered}
H\left(\mathbb{X},\left|\Psi_{\text {sep }}\right\rangle\right)+H\left(\mathbb{Y},\left|\Psi_{\text {sep }}\right\rangle\right)= \\
\mathcal{H}_{2}\left(|\cos \alpha \cos \beta|^{2}+|\sin \alpha \sin \beta|^{2}\right) \\
+\mathcal{H}_{2}[(1-\sin \delta \sin \gamma \sin (2 \alpha) \sin (2 \beta)) / 2] .
\end{gathered}
$$


We are interested in the minimum value achievable by this four-parameter function. The analysis is simplified by the fact that $\mathcal{H}_{2}(x)$ is a decreasing function of $|1-2 x|$. Hence, for any $\alpha, \beta \in[0, \pi / 2]$ the right-hand-side of Eq. (12) reaches its minimum for $\delta, \gamma= \pm \pi / 2$. Assigning these values for $\delta$ and $\gamma$, Eq. (12) can then be shown to have minimum equal to $\ln 2$ (e.g. see Fig. 11), concluding the proof [20].

Entropic uncertainty relations can be derived for more than two observables at a time 16]. In order to exploit this effect, we introduce a third observable, $\hat{Z} \equiv \sigma_{a}^{(3)} \otimes \sigma_{b}^{(3)}$ and derive a separability condition which is independent from Eq. (7). On one hand, since $\hat{Z}$ commutes with the operators $\hat{X}, \hat{Y}$ of Eq. (5), for a generic state $\rho$ we have

$$
H(\mathbb{X}, \rho)+H(\mathbb{Y}, \rho)+H(\mathbb{Z}, \rho) \geqslant 0,
$$

where the equality is achieved on the Bell states of Eq. (6). On the other hand, when the quantity on the left-hand-side of Eq. (13) is evaluated a separable state it obeys to the following inequality (see App. A2 for the derivation),

$$
H\left(\mathbb{X}, \rho_{\text {sep }}\right)+H\left(\mathbb{Y}, \rho_{\text {sep }}\right)+H\left(\mathbb{Z}, \rho_{\text {sep }}\right) \geqslant 2 \ln 2 .
$$

This relation provides a weaker separability condition, i.e. a more sensitive entanglement criteria, than Eq. (7). In fact, since each of the operators $\hat{X}, \hat{Y}$ and $\hat{Z}$ has only two orthogonal eigenspaces, the quantities $H\left(\mathbb{X}, \rho_{\text {sep }}\right)$, $H\left(\mathbb{Y}, \rho_{\text {sep }}\right)$ and $H\left(\mathbb{Z}, \rho_{\text {sep }}\right)$ are always smaller than $\ln 2$. Using this property it is straightforward to show that Eq. (14) implies Eq. (77). This means that if a state $\rho$ is entangled according to Eq. (7) (i.e. if $\rho$ violates such inequality), then it is also entangled according to Eq. (14) (i.e. $\rho$ violates also this inequality). The opposite, however, is not true: entangled states that satisfy the inequality (7) but not the inequality (14) exist (e.g. see the case discussed in Sec. [ID).

\section{B. Operators diagonal in the Bell basis}

We have seen that the operators $\hat{X}, \hat{Y}$ and $\hat{Z}$ form a filtering system, which is partially permeable to entangled states, but which does not allow any separable states to pass without paying a $2 \ln 2$ fee in uncertainty. A better insight on this property can be obtained by analyzing the decomposition of these observables in the Bell basis (6),

$$
\begin{aligned}
\hat{X} & \equiv\left|\Psi_{1}\right\rangle\left\langle\Psi_{1}|+| \Psi_{2}\right\rangle\left\langle\Psi_{2}|-| \Psi_{3}\right\rangle\left\langle\Psi_{3}|-| \Psi_{4}\right\rangle\left\langle\Psi_{4}\right| \\
\hat{Y} & \equiv-\left|\Psi_{1}\right\rangle\left\langle\Psi_{1}|+| \Psi_{2}\right\rangle\left\langle\Psi_{2}|+| \Psi_{3}\right\rangle\left\langle\Psi_{3}|-| \Psi_{4}\right\rangle\left\langle\Psi_{4}\right| \\
\hat{Z} & \equiv\left|\Psi_{1}\right\rangle\left\langle\Psi_{1}|-| \Psi_{2}\right\rangle\left\langle\Psi_{2}|+| \Psi_{3}\right\rangle\left\langle\Psi_{3}|-| \Psi_{4}\right\rangle\left\langle\Psi_{4}\right| .
\end{aligned}
$$

\begin{tabular}{|c|c|c|c|c|c|c|c|c|c|}
\hline & & & & & & $\left|\Psi_{1}\right\rangle$ & $\left|\Psi_{2}\right\rangle$ & $\left|\Psi_{3}\right\rangle$ & $\left.\Psi_{4}\right\rangle$ \\
\hline & $\left|\Psi_{1}\right\rangle$ & $\left|\Psi_{2}\right\rangle$ & $\left|\Psi_{3}\right\rangle$ & $\left|\Psi_{4}\right\rangle$ & $\hat{X}_{1}^{(1,1,2)}$ & 0 & 0 & +1 & -1 \\
\hline$\hat{X}_{1}^{(1,3)}$ & +1 & -1 & -1 & -1 & $\hat{X}_{2}^{(1,1,2)}$ & 0 & +1 & 0 & -1 \\
\hline$\hat{X}_{2}^{(1,3)}$ & -1 & +1 & -1 & -1 & $\hat{X}_{3}^{(1,1,2)}$ & 0 & +1 & -1 & 0 \\
\hline$\hat{X}_{3}^{(1,3)}$ & -1 & -1 & +1 & -1 & $\hat{X}_{4}^{(1,1,2)}$ & +1 & 0 & 0 & -1 \\
\hline$\hat{X}_{4}^{(1,3)}$ & -1 & -1 & -1 & +1 & $\hat{X}_{5}^{(1,1,2)}$ & +1 & 0 & -1 & 0 \\
\hline & & & & & $\hat{X}_{6}^{(1,1,2)}$ & +1 & -1 & 0 & 0 \\
\hline
\end{tabular}

Each of the above operators identifies two couples of Bell states associated with the +1 and -1 eigenvalue respectively. By measuring $\hat{X}$, for instance, we can distinguish
TABLE I: On the left: Spectral decomposition of a set of operators $\hat{X}_{j}^{(1,3)}$ with $j=1, \cdots, 4$ that distinguish only one of the Bell states of Eq. (6) from the other three. Notice that there are at most four of these observables that differ topologically from each other. On the right: Spectral decomposition of a set of operators $\hat{X}_{j}^{(1,1,2)}$ with $j=1, \cdots, 6$ that divide the Bell state into a group of two and two groups of one states. There are at most six of them that are topologically not equivalent. In both the tables, the value in the $j$ th row and $v$ th column is the eigenvalue of the $j$ th operator associated with $\left|\Psi_{v}\right\rangle$.

the first two Bell states from the last two, but we cannot distinguish $\left|\Psi_{1}\right\rangle$ from $\left|\Psi_{2}\right\rangle$ or $\left|\Psi_{3}\right\rangle$ from $\left|\Psi_{4}\right\rangle$. Moreover $\hat{X}, \hat{Y}$ and $\hat{Z}$ identify different subsets of the Bell basis and hence form a set of topologically distinguishable observables.

Consider now the separable state $|00\rangle=\left(\left|\Psi_{1}\right\rangle+\right.$ $\left.\left|\Psi_{2}\right\rangle\right) / \sqrt{2}$. On one hand, since this is an eigenstate of $\hat{X}$, the uncertainty of such observable nullifies. On the other hand, since $|00\rangle$ is a uniform superposition of distinguishable eigenstates of $\hat{Y}$, the measurement of this observable gives +1 or -1 with probability $1 / 2$ and produces hence one bit $(\ln 2)$ of entropic uncertainty as requested by the inequality (7). The same is true also for $\hat{Z}$ : the measurement of this observable on $|00\rangle$ produces one bit of uncertainty in agreement with Eq. (14).

One way to generalize the above result is to design different filtering strategies by selecting appropriate sets of nonlocal operators. In Table \ we present two examples that employ observables diagonal in the Bell basis (6). In the first case, we use the operators $\hat{X}_{j}^{(1,3)}$ that individually distinguish one particular Bell state from the remaining three, but not these last from each other. As in the case of the operators $\hat{X}, \hat{Y}$ and $\hat{Z}$, the Bell states (6) are the only ones that can nullify the entropic uncertainties of all the $\hat{X}_{j}^{(1,3)}$. One can use these operators to derive a separable condition analogous to (14). In fact, consider again the state $|00\rangle$ : in this case it diagonalizes $\hat{X}_{3}^{(1,3)}$ and $\hat{X}_{4}^{(1,3)}$ but, according to Table $\llbracket$ produces one bit of uncertainty when measured with $\hat{X}_{1}^{(1,3)}$ or $\hat{X}_{2}^{(1,3)}$. As discussed in the App. A3 this result can be formalized by showing that for any separable state $\rho_{\text {sep }}$ one has

$$
\sum_{j=1}^{4} H\left(\mathbb{X}_{j}^{(1,3)}, \rho_{\text {sep }}\right) \geqslant 2 \ln 2 .
$$

Analogously to the case of the operators (15), also here it is possible to identify a minimum number of elements 
necessary to produce a nontrivial minimal uncertainty for separable states. In the case of the operators $\hat{X}, \hat{Y}$ and $\hat{Z}$ such number is two: in fact, there are separable states that diagonalize at least one of these observables (see the example of $|00\rangle$ discussed above) but there are no separable states that diagonalize two of them (see Eq. (7)). In the case of the operators $\hat{X}_{j}^{(1,3)}$ such a minimum number is three since there are examples of separable states that diagonalize any two of them.

The same considerations applies also to the operators $\hat{X}_{j}^{(1,1,2)}$ of Table \which divide the Bell basis into two subgroups of one state and one subgroup of two states. In this case we have (see App. A4)

$$
\sum_{j=1}^{6} H\left(\mathbb{X}_{j}^{(1,1,2)}, \rho_{\text {sep }}\right) \geqslant 5 \ln 2
$$

since separable states can diagonalize only one of the $\hat{X}_{j}^{(1,1,2)}$ at the time.

The final example we consider here is given by one single observable which assigns a different eigenvalue to each of the four Bell states, e.g.

$$
\begin{aligned}
\hat{X}^{(1,1,1,1) \equiv} & \left|\Psi_{1}\right\rangle\left\langle\Psi_{1}|+2| \Psi_{2}\right\rangle\left\langle\Psi_{2}\right| \\
& +3\left|\Psi_{3}\right\rangle\left\langle\Psi_{3}|+4| \Psi_{4}\right\rangle\left\langle\Psi_{4}\right| .
\end{aligned}
$$

To form a separable state we need to superimpose at least two different vectors of the Bell basis (see App. A 1). Hence, the measurement of $\hat{X}^{(1,1,1,1)}$ on $\rho_{\text {sep }}$ produces at least one bit of uncertainty, i.e.

$$
H\left(\mathbb{X}^{(1,1,1,1)}, \rho_{\text {sep }}\right) \geqslant \ln 2 .
$$

This result is analytically proved in App. A1 and, analogously to Eqs. (16) and (17), gives a separability condition for a two-qubit system. In Sec. III we will show how to generalize the above inequalities to bipartite systems of higher dimension.

\section{Noncommuting observables}

An alternative example of entropic separability conditions for a two-qubit system, is provided by the three orthogonal components of the total spin of the system,

$$
\begin{aligned}
& \hat{S}_{1} \equiv \sigma_{a}^{(1)} \otimes \mathbb{1}_{b}+\mathbb{1}_{a} \otimes \sigma_{b}^{(1)} \\
& \hat{S}_{2} \equiv \sigma_{a}^{(2)} \otimes \mathbb{1}_{b}+\mathbb{1}_{a} \otimes \sigma_{b}^{(2)} \\
& \hat{S}_{3} \equiv \sigma_{a}^{(3)} \otimes \mathbb{1}_{b}+\mathbb{1}_{a} \otimes \sigma_{b}^{(3)}
\end{aligned}
$$

where $\mathbb{1}_{s}$ is the identity operator on the qubit $s$. These observables do not commute with each other but the sum of their entropic uncertainties is nullified by $\left|\Psi_{4}\right\rangle$ of (6) (this is the singlet state which is an eigenvector of the total spin). In other words, for $\rho$ generic we have again

$$
\sum_{j=1}^{3} H\left(\mathbb{S}_{j}, \rho\right) \geqslant 0 \text {. }
$$

As in the previous cases, however, if the state $\rho$ is separable, a nontrivial constraint on the sum of the entropic uncertainties applies. This happens because the three components of the total spin do not share any common separable eigenstate. In particular in this case one can verify that for any $\rho_{\text {sep }}$ separable (see App. A5),

$$
\sum_{j=1}^{3} H\left(\mathbb{S}_{j}, \rho_{\text {sep }}\right) \geqslant 3 \ln 2 .
$$

\section{Comparison with existing entanglement criteria}

Here we analyze the sensitivity in detecting the presence of entanglement of the inequalities derived in the previous sections. Following the suggestion of [10] we consider the set of Werner states

$$
w_{p} \equiv \frac{1-p}{4} \mathbb{1}_{a} \otimes \mathbb{1}_{b}+p\left|\Psi_{4}\right\rangle\left\langle\Psi_{4}\right|
$$

where $\left|\Psi_{4}\right\rangle$ is the state defined in (6) and $p \in[0,1]$. The density matrices $w_{p}$ are separable if and only if $p \leqslant 1 / 3$ [21]. To test the relations (7) and (14) we need to evaluate the entropic uncertainties defined in Eq. (2) for each one of the operators $\sigma_{a}^{(j)} \otimes \sigma_{b}^{(j)}$, with $j=1,2,3$. However, since the states $w_{p}$ are rotationally invariant, these quantities are identical and it is sufficient to evaluate only one of them. In particular consider $\hat{X}=\sigma_{a}^{(1)} \otimes \sigma_{b}^{(1)}$. The projector operators in the eigenspaces of this observable are $\mathbb{X}_{+1} \equiv|00\rangle\langle 00|+| 11\rangle\langle 11|$ and $\mathbb{X}_{-1} \equiv|01\rangle\langle 01|+| 10\rangle\langle 10|$, so that the probabilities $P_{k}$ of Eq. (2) for the state $w_{p}$ are $P_{ \pm}=(1 \pm p) / 2$. Consequently, we have

$$
\begin{aligned}
& H\left(\mathbb{X}, w_{p}\right)+H\left(\mathbb{Y}, w_{p}\right)=2 \mathcal{H}_{2}\left(\frac{1+p}{2}\right) \\
& H\left(\mathbb{X}, w_{p}\right)+H\left(\mathbb{Y}, w_{p}\right)+H\left(\mathbb{Z}, w_{p}\right)=3 \mathcal{H}_{2}\left(\frac{1+p}{2}\right),
\end{aligned}
$$

with $\mathcal{H}_{2}(x)$ the binary entropy function (11). According to our analysis we can conclude that the state $w_{p}$ is entangled if Eq. (24) or 25) violates the lower bounds established by Eqs. (77), (14), respectively. In the first case, this happens for $p>0.78$, while in the second case it is sufficient to have $p>0.65$. [This is in agreement with the fact Eq. (14) is a weaker separability condition than Eq. (7)]. The same analysis can be repeated for the separability conditions (16), (17) and (19) yielding respectively,

$$
\begin{aligned}
& 3 \mathcal{H}_{2}\left(\frac{1-p}{4}\right)+\mathcal{H}_{2}\left(\frac{1+3 p}{4}\right) \geqslant 2 \ln 2 \\
& 3 \mathcal{H}_{2}\left(\frac{1+p}{2}\right)+3 \mathcal{F}\left(\frac{1+3 p}{4}\right)+9 \mathcal{F}\left(\frac{1-p}{4}\right) \geqslant 5 \ln 2 \\
& 3 \mathcal{F}\left(\frac{1-p}{4}\right)+\mathcal{F}\left(\frac{1+3 p}{4}\right) \geqslant \ln 2,
\end{aligned}
$$


where $\mathcal{F}(x)=-x \ln x$. The fist of these relations is violated for $p>0.68$, the second for $p>0.72$ and the last for $p>0.74$. Hence the separability condition (14) still provides the best criterion. However, we cannot conclude that (14) is, in general, a more sensitive criterion than (16), (17) and (19). As a matter of fact, there is not a clear general ordering between these criteria: the best performance of (14) in this case is due to the symmetry of the states (23).

Let us now analyze the noncommuting set of observables analyzed in Sec. (IIC). Such operators have only $\left|\Psi_{4}\right\rangle$ as common eigenvector and hence we expect the separability condition (22) to be better suited to detect the presence of entanglement in $w_{p}$. In this case the entropic uncertainty is

$$
\sum_{j=1}^{3} H\left(\mathbb{S}_{j}, w_{p}\right)=3 \mathcal{F}\left(\frac{1+p}{2}\right)+6 \mathcal{F}\left(\frac{1-p}{4}\right)
$$

with $\mathcal{F}(x)$ defined as in Eq. (26). By comparing this function with the separability condition (22), we can establish that $w_{p}$ is entangled for $p>0.55$. As expected the entanglement criterion based on this relation is hence able to recognize more entangled states than the previous ones. Nevertheless, our best example is still not able to pinpoint the threshold $p=1 / 3$ as, instead, the criterion proposed in [10] does, or even achieve the threshold $p=1 / 2$ as the criterion proposed in 8,22$]$. Of course this can be just a consequence of the choice of the operators we have assumed in developing the entropic separability conditions proposed here: refining this choice the method might achieve better performances.

\section{SEPARABILITY CONDITIONS FOR $d \times d$ BIPARTITE SYSTEMS.}

In this section we discuss the entropic separability conditions for $d \times d$ bipartite systems $\mathcal{S}$ where the two subsystems $\mathcal{A}$ and $\mathcal{B}$ are associated with Hilbert spaces of equal dimension $d \geqslant 2$. The situation is not different, in principle, from the qubit case analyzed in Sec. III Also here one needs to define a collection of nonlocal operators $\left\{\hat{X}_{j}\right\}$ of $\mathcal{S}$ which do not have any common separable eigenvector. For at least some of these sets one expects the global entropic uncertainty to have a nontrivial lower bound when evaluated over the states $\rho_{\text {sep }}$ of Eq. (4). In other words, define

$$
\begin{aligned}
E & \equiv \min _{\rho} \sum_{j=1}^{J} H\left(\mathbb{X}_{j}, \rho\right) \\
E_{\text {sep }} & \equiv \min _{\rho_{\text {sep }}} \sum_{j=1}^{J} H\left(\mathbb{X}_{j}, \rho_{\text {sep }}\right),
\end{aligned}
$$

where the first minimization is performed for all $\rho$ of $\mathcal{S}$ and the second only on the separable states (4) of the system. $E_{\text {sep }}$ is clearly greater or equal to $E$. Here, however, we are looking for sets of operators that have a strictly positive gap $\Delta$ between these quantities, i.e.

$$
\Delta \equiv E_{\text {sep }}-E>0 \text {. }
$$

For any $\left\{\hat{X}_{j}\right\}$ for which this inequality holds we have a separability condition on $\mathcal{S}$. In general the calculation of $\Delta$ is nontrivial since it requires to find global minima of entropic quantities [23]. In the following section we will present a partial solution of this problem by focusing on a special class of commuting operators $\hat{X}_{j}$ which are diagonal in a maximally entangled basis of $\mathcal{S}$.

\section{A. Bell sets}

A maximally entangled state $\left|\Psi_{\text {bell }}\right\rangle$ of $\mathcal{S}$ is a state that has maximally mixed reduced density matrix, e.g.

$$
\rho_{a} \equiv \operatorname{Tr}_{b}\left[\left|\Psi_{\text {bell }}\right\rangle\left\langle\Psi_{\text {bell }}\right|\right]=\mathbb{1}_{a} / d
$$

where $\operatorname{Tr}_{a}[\cdots]$ is the partial trace over the subsystem $\mathcal{A}$. The Bell states (6) are an example of maximally entangled states for a two-qubit system. Consider an orthonormal basis $\left\{\left|\Psi_{v}\right\rangle\right\}$ composed by these particular vectors. As in Sec. IIB we will consider sets of Bell operators, i.e. ensembles of nonlocal observables which are diagonal with respect to $\left\{\left|\Psi_{v}\right\rangle\right\}$ and divide the elements of such basis into subgroups of degenerate eigenvectors. For instance, the Bell set $\left\{\hat{X}_{j}^{\left(M_{1}, \cdots, M_{K}\right)}\right\}$ contains all the topologically distinguishable observables which have $K \leqslant d^{2}$ distinct eigenvalues of degeneracies $M_{1}, \cdots, M_{K}$. Clearly the absolute minimum $E$ of Eq. (28) of each of these ensembles is zero (their operators commutes): thus, to establish if a Bell set can be used to derive a separable condition it is sufficient to evaluate the quantity $E_{\text {sep }}$ of Eq. (29). This problem is still difficult to solve, even though in many case it is possible to heuristically guess the right answer. In the following we will analyze the simplest of these Bell sets which are respectively the generalization to the case $d \geqslant 2$ of the operators $\hat{X}^{(1,1,1,1)}$ and $\hat{X}_{j}^{(1,3)}$ of Sec. (IIB): at least in these cases it is relatively easy to derive analytically the solution.

\section{First case}

Consider the nonlocal operator

$$
\hat{X}^{(1, \cdots, 1)} \equiv \sum_{v=1}^{d^{2}} v\left|\Psi_{v}\right\rangle\left\langle\Psi_{v}\right|
$$

This is the simplest example of a Bell set: it contains a single element that distinguishes all the elements of the maximally entangled basis from each other. As shown in App. B the projection probability of any separable state 
$\rho_{\text {sep }}$ on a generic vector of the maximally entangled basis cannot be greater than $1 / d$, i.e.

$$
Q_{v} \equiv\left\langle\Psi_{v}\left|\rho_{\text {sep }}\right| \Psi_{v}\right\rangle \leqslant 1 / d
$$

for any $v=1, \cdots, d^{2}$. Hence from the definitions (2) and (32) we have,

$$
H\left(\mathbb{X}^{(1, \cdots, 1)}, \rho_{\text {sep }}\right) \equiv-\sum_{v=1}^{d^{2}} Q_{v} \ln Q_{v} \geqslant \ln d .
$$

This, together with the fact that $E=0$, shows that the gap $\Delta$ of Eq. (30) is $>0$. Thus, Eq. (34) provides an entanglement criterion for $d \times d$ bipartite systems. For $d=2$ it reduces to Eq. (19).

\section{Second case}

Consider now the Bell set composed by the following $d^{2}$ topologically distinct operators

$$
\hat{X}_{v}^{\left(1, d^{2}-1\right)} \equiv 2\left|\Psi_{v}\right\rangle\left\langle\Psi_{v}\right|-\mathbb{1}
$$

where $v=1, \cdots, d^{2}$ and $\mathbb{1}$ is the identity operator of $\mathcal{S}$. These observables are the generalization for $d \geqslant 2$ of the operators $\hat{X}_{j}^{(1,3)}$ defined in Table凹 each of them divides the maximally entangled basis $\left\{\left|\Psi_{v}\right\rangle\right\}$ into two groups, the first containing the eigenvector associated with the eigenvalue +1 and the second containing the $d^{2}-1$ eigenvectors associated with the eigenvalue -1 . We expect $E_{\text {sep }}$ of Eq. (29) to be strictly positive. Roughly speaking, since any separable state $\left|\Psi_{\text {sep }}\right\rangle$ must be a superposition of at least $d$ orthogonal maximally entangled states (see Eq. (38) below), one should have an average uncertainty of $\mathcal{H}_{2}(1 / d)$ from every $\hat{X}_{j}^{\left(1, d^{2}-1\right)}$ not diagonalized by $\left|\Psi_{\text {sep }}\right\rangle$. Moreover, the same argument can be used to show that the minimum number of non diagonalized operators must of the order of $d$. [In fact, given a collection of $d$ basis elements, the maximum number of $\hat{X}_{j}^{\left(1, d^{2}-1\right)}$ that assign the eigenvalue +1 to one of such vectors is $d$ ]. In other words, it is reasonable to assume that the following inequality applies to any separable state $\rho_{\text {sep }}$ of the system,

$$
\sum_{j=1}^{d^{2}} H\left(\mathbb{X}_{j}^{\left(1, d^{2}-1\right)}, \rho_{\text {sep }}\right) \geqslant d \mathcal{H}_{2}(1 / d),
$$

where $\mathcal{H}_{2}$ is the binary entropy function of Eq. (111). [Notice that for $d=2$ the above expression reduces to the inequality (16)]. To prove Eq. (36) we first notice that the entropic uncertainty of the operator $\hat{X}_{j}^{\left(1, d^{2}-1\right)}$ can be expressed as

$$
H\left(\mathbb{X}_{j}^{\left(1, d^{2}-1\right)}, \rho_{\text {sep }}\right)=\mathcal{H}_{2}\left(Q_{j}\right) .
$$

Moreover, given a separable state $\rho_{\text {sep }}$, there must be at least $d$ elements of the maximally entangled basis $\left\{\left|\Psi_{v}\right\rangle\right\}$ that have projection probabilities $Q_{v}>0$. In fact, let $r$ be the number of such elements, then from the normalization condition of $Q_{v}$ and from the property (33) we have

$$
1=\sum_{v=1}^{d^{2}} Q_{v} \leqslant r / d
$$

Ordering the $Q_{v}$ in decreasing order, we can hence write the entropic uncertainty of this set of operators as follows

$$
\sum_{j=1}^{d^{2}} H\left(\mathbb{X}_{j}^{\left(1, d^{2}-1\right)}, \rho_{\text {sep }}\right)=\sum_{j=1}^{d} \mathcal{H}_{2}\left(Q_{j}\right)+\sum_{j=d+1}^{d^{2}} \mathcal{H}_{2}\left(Q_{j}\right)
$$

Every $Q_{j}$ in the first summation is bigger than the $Q_{j}$ in the second summation. We can hence use the property iii) of the binary entropy given in App. @ to derive a lower bound for (39). Namely, according to Eq. (A2) some positive quantities can be subtracted from each of the arguments of the binary entropies of the second summation and added to the arguments of the binary entropy of the first summation. Equations (33) and (38) guarantee that these quantities can be chosen to transform into $1 / d$ all the arguments in the first summation, nullifying in the meantime all the arguments in the second one. This concludes the proof because we have generated a lower bound of (39) made of $d$ binary entropies evaluated in $1 / d$.

\section{CONCLUSIONS}

The inequalities presented in this paper are examples of how one can construct separability conditions for bipartite systems starting from entropic uncertainty relations. They derive from the fact that the common eigenstates of some nonlocal operators are entangled. As in the case of separability conditions obtained from the Heisenberg-like uncertainty relation [6, 7, 8, 9, 10], this property can be exploited to impose bounds on the minimum indetermination that can be achieved when measuring simultaneously these operators on separable states. A detailed analysis of this procedure has been provided in the case of two-qubit systems. The main problem in deriving entropic separability conditions resides with the fact that one has to solve a (constrained) minimization of a concave function (the Shannon entropy). This in general is a difficult task even in the case of a commuting set of observables. A possible solution of this problem could be obtained, for instance, by replacing the Shannon entropy with the Rényi entropies which are sometime easier to handle. At this stage of the investigation it is not yet clear if entropic separability conditions are competitive with respect to other strategies (when they are available): in the examples discussed in the paper we have found evidences of the contrary. However, since the 
method proposed here relays on measurable quantities (the probabilities of finding the state in the eigenspaces associated with certain operators) it can be a useful tool in characterizing the presence of entanglement in many experimental context.

\section{APPENDIX A: DERIVATION OF THE SEPARABILITY CONDITIONS FOR QUBITS}

In this Appendix we prove the inequalities introduced in Sec. II which provide the separability conditions for the two-qubit system.

In the derivation we will use of the following properties of the binary entropy of Eq. (11):

i) For $x \in[0,1]$ the function $\mathcal{H}_{2}(x)$ is decreasing in $|1-2 x|,[19]$.

ii) For $x, y \in[0,1]$ and $x+y \leqslant 1$ one has

$$
\mathcal{H}_{2}(x)+\mathcal{H}_{2}(y) \geqslant \mathcal{H}_{2}(x+y) \text {. }
$$

This can be seen by studying the dependence on $y$ of the difference between the left-hand-side and the right-hand-side of Eq. (A1).

iii) The previous relation can be generalized to show that for any $x, y$ and $z \in[0,1]$ with $1-y \geqslant x \geqslant$ $y \geqslant z$, we have

$$
\mathcal{H}_{2}(x)+\mathcal{H}_{2}(y) \geqslant \mathcal{H}_{2}(x+z)+\mathcal{H}_{2}(y-z) .
$$

For $z=0$ this is a trivial identity. For $z$ positive the above inequality can be obtained by observing that the first derivative in $z$ of the difference between the left-hand-side and the right-hand-side of Eq. (A2) is positive for all $z$ in the domain.

As in the case of Eq. (7), we will use the concavity of the Shannon entropy [19] to limit the analysis to the pure separable states $\left|\Psi_{\text {sep }}\right\rangle$ of Eq. (8): if the inequalities (14), (16), (17) and (19) apply for this class of separable states, then they hold also for any mixed separable states of the form (4). The projection probabilities $Q_{v} \equiv\left|\left\langle\Psi_{\text {sep }} \mid \Psi_{v}\right\rangle\right|^{2}$ of $\left|\Psi_{\text {sep }}\right\rangle$ on the Bell vectors of Eq. (6) will be expressed as

$$
\begin{array}{ll}
Q_{1}=\left(q_{0}+q_{1}\right) / 2 & Q_{3}=\left(1-q_{0}+q_{2}\right) / 2 \\
Q_{2}=\left(q_{0}-q_{1}\right) / 2 & Q_{4}=\left(1-q_{0}-q_{2}\right) / 2
\end{array}
$$

where $q_{1}=q \cos (\delta+\gamma), q_{2}=q \cos (\delta-\gamma)$ and

$$
\begin{aligned}
q_{0} & =|\cos \alpha \cos \beta|^{2}+|\sin \alpha \sin \beta|^{2} \\
q & =2 \cos \alpha \cos \beta \sin \alpha \sin \beta .
\end{aligned}
$$

These quantities satisfy the relation $q_{0} \pm q=\cos ^{2}(\alpha \mp \beta)$ which implies

$$
\left|q_{1}\right|,\left|q_{2}\right| \leqslant|q| \leqslant \min \left[q_{0}, 1-q_{0}\right],
$$

and $Q_{v} \leqslant 1 / 2$ for all $v=1, \cdots, 4$.

\section{Derivation of Eq. (19)}

We start from this separability condition because it is the easiest to derive. From the definitions (2) and (18) we have

$$
H\left(\mathbb{X}^{(1,1,1,1)},\left|\Psi_{s e p}\right\rangle\right)=-\sum_{v=1}^{4} Q_{v} \ln Q_{v}
$$

with $Q_{v}$ defined in (A3). The inequality (19) then is a consequence of $Q_{v} \leqslant 1 / 2$, i.e. $-\ln Q_{v} \geqslant-\ln (1 / 2)$.

\section{Derivation of Eq. (14)}

The spectral decompositions (15) allow us to express the entropic uncertainties of the operators $\hat{X}, \hat{Y}$ and $\hat{Z}$ on the state $\left|\Psi_{\text {sep }}\right\rangle$ in terms of the probabilities $Q_{v}$ of Eq. (A3), i.e.

$$
\begin{aligned}
& \sum_{j=1}^{3} H\left(\mathbb{X}_{j}^{(2,2)},\left|\Psi_{\text {sep }}\right\rangle\right)=\mathcal{H}_{2}\left(q_{0}\right) \\
& \quad+\mathcal{H}_{2}\left(\frac{1+q_{2}-q_{1}}{2}\right)+\mathcal{H}_{2}\left(\frac{1+q_{1}+q_{2}}{2}\right)
\end{aligned}
$$

where, following the notation introduced in Sec. IIB we defined $\hat{X}_{1}^{(2,2)} \equiv \hat{X}, \hat{X}_{2}^{(2,2)} \equiv \hat{Y}$ and $\hat{X}_{3}^{(2,2)} \equiv \hat{Z}$. Using the properties i) and ii) of $\mathcal{H}_{2}$ it is possible to derive a lower bound for the above expression. Consider for instance the case $q_{2} \geqslant q_{1} \geqslant 0$. According to ii) we obtain a lower bound for A7 by subtracting $\left(q_{2}-q_{1}\right) / 2$ from the argument of the second binary entropy term on the right-hand-side of Eq. A7 and by adding it to the argument of the third binary entropy term, i.e.

$$
\begin{gathered}
\mathcal{H}_{2}\left(\frac{1+q_{2}-q_{1}}{2}\right)+\mathcal{H}_{2}\left(\frac{1+q_{1}+q_{2}}{2}\right) \\
\geqslant \mathcal{H}_{2}(1 / 2)+\mathcal{H}_{2}\left(1 / 2+q_{2}\right) .
\end{gathered}
$$

Using the symmetries of Eq. (A77) and $\mathcal{H}_{2}(x)$ it is easy to show that for $q_{1}, q_{2}$ generic the above inequality still applies if we replace $q_{2}$ with $\max \left(\left|q_{1}\right|,\left|q_{2}\right|\right)$ in the righthand-side term. By replacing $q_{j}$ with $\min \left[q_{0}, 1-q_{0}\right]$ in the previous expression, the property i) of binary entropy and Eq. (A5) allow then to establish the following inequality,

$$
\sum_{j=1}^{3} H\left(\mathbb{X}_{j}^{(2,2)},\left|\Psi_{\text {sep }}\right\rangle\right) \geqslant \ln 2+\mathcal{G}\left(q_{0}\right),
$$

where, for $q_{0} \in[0,1]$,

$$
\mathcal{G}\left(q_{0}\right) \equiv \mathcal{H}_{2}\left(q_{0}\right)+\mathcal{H}_{2}\left(1 / 2+\min \left[q_{0}, 1-q_{0}\right]\right) .
$$

The thesis (14) is hence a consequence of the fact $\mathcal{G}\left(q_{0}\right)$ has absolute minimum equal to $\ln 2$ for $q_{0}=1 / 2$. [This last property can be verified by studying the first derivative of $\left.\mathcal{G}\left(q_{0}\right)\right]$. 


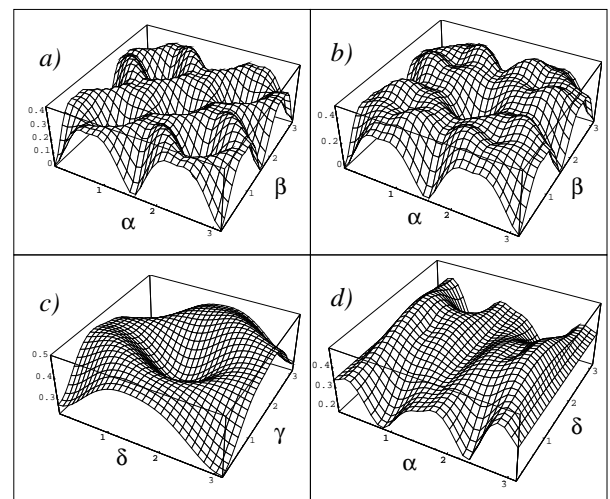

FIG. 2: Plot of the difference between the left-hand-side and the right-hand-side of Eq. (22) for the state $\left|\Psi_{\text {sep }}\right\rangle$ of Eq. (8): this function is positive. In a) $\delta=\gamma=\pi$; in b) $\delta=-\pi / 2$, and $\gamma=\pi$; in c) $\alpha=-\pi / 3$, and $\beta=-\pi / 5$; in $d$ ) $\beta=\pi / 3$, and $\gamma=0$.

\section{Derivation of Eq. (16)}

The derivation of the separability condition (16) proceeds as in the previous section. First we express the entropic uncertainty relation of the operators $\hat{X}_{j}^{(1,3)}$ in terms of the probabilities $Q_{v}$ of Eq. A33). In this case, according to the spectral decomposition of Table \we have

$$
\sum_{j=1}^{4} H\left(\mathbb{X}_{j}^{(1,3)},\left|\Psi_{s e p}\right\rangle\right)=\sum_{j=1}^{4} \mathcal{H}_{2}\left(Q_{v}\right) .
$$

From the definition (11) we can write the first two contribution on the right-hand-side of Eq. A11) as

$$
\begin{aligned}
& \mathcal{H}_{2}\left(Q_{1}\right)+\mathcal{H}_{2}\left(Q_{2}\right)=-q_{0} \ln q_{0}-\left(2-q_{0}\right) \ln \left(2-q_{0}\right) \\
& +q_{0} \mathcal{H}_{2}\left(\frac{q_{0}+q_{1}}{2 q_{0}}\right)+\left(2-q_{0}\right) \mathcal{H}_{2}\left(\frac{2-q_{0}+q_{1}}{2\left(2-q_{0}\right)}\right)
\end{aligned}
$$

which, according to the property i) of the binary entropy, reaches its minimum value when $\left|q_{1}\right|$ reaches its maximum $\min \left[q_{0}, 1-q_{0}\right]$. Analogously we can show that $\mathcal{H}_{2}\left(Q_{3}\right)+\mathcal{H}_{2}\left(Q_{4}\right)$ has its minimum for $\left|q_{2}\right|=\min \left[q_{0}, 1-\right.$ $\left.q_{0}\right]$. Together these relations provide the following lower bound of the total entropic uncertainty (A11)

$$
\sum_{j=1}^{4} H\left(\mathbb{X}_{j}^{(1,3)},\left|\Psi_{s e p}\right\rangle\right) \geqslant \ln 2+\mathcal{G}\left(q_{0}\right),
$$

where $\mathcal{G}\left(q_{0}\right)$ is the function defined in Eq. A10. The thesis (16) follows once again from the fact that the ab- solute minimum of $\mathcal{G}\left(q_{0}\right)$ is $\ln 2$.

\section{Derivation of Eq. (17)}

The separability condition (17) is slightly more demanding to prove than the previous ones. Here, according to Table【 we have six different observables $\hat{X}_{j}^{(1,1,2)}$, each with three distinct eigenvalues: the two times degenerate eigenvalue 0 and the non degenerate eigenvalues \pm 1 . For instance, for $j=1$ we have

$$
\begin{aligned}
H\left(\mathbb{X}_{1}^{(1,1,2)},\left|\Psi_{\text {sep }}\right\rangle\right)= & -\left(Q_{1}+Q_{2}\right) \ln \left(Q_{1}+Q_{2}\right) \\
& -Q_{3} \ln Q_{3}-Q_{4} \ln Q_{4} .(\mathrm{A}
\end{aligned}
$$

Some elementary simplifications allow to express the total entropic uncertainty as the sum of three contributions

$$
\sum_{j=1}^{6} H\left(\mathbb{X}_{1}^{(1,1,2)},\left|\Psi_{s e p}\right\rangle\right)=f_{0}+f_{1}+f_{2}
$$

where

$$
\begin{aligned}
f_{0} & \equiv \mathcal{H}_{2}\left(q_{0}\right)+\mathcal{H}_{2}\left(\frac{1+q_{2}-q_{1}}{2}\right)+\mathcal{H}_{2}\left(\frac{1+q_{1}+q_{2}}{2}\right) \\
f_{1} & \equiv 3 q_{0} \mathcal{H}_{2}\left(\frac{q_{0}+q_{1}}{2 q_{0}}\right)-3 q_{0} \ln q_{0} \\
f_{2} & \equiv 3\left(1-q_{0}\right) \mathcal{H}_{2}\left(\frac{1-q_{0}+q_{2}}{2\left(1-q_{0}\right)}\right)-3\left(1-q_{0}\right) \ln \left(1-q_{0}\right) .
\end{aligned}
$$

We notice that $f_{0}$ is the function given in Eq. A77) and that, according to the analysis of App. A2 it is always greater than or equal to $2 \ln 2$. On the other hand, using the property i) of the binary entropy, we observe that $f_{1}$ and $f_{2}$ are decreasing functions of $\left|q_{1}\right|$ and $\left|q_{2}\right|$, respectively. Hence using Eq. A5 we obtain

$$
f_{1}+f_{2} \geqslant 3 \ln 2+\frac{3}{2} \mathcal{H}_{2}\left(2 \min \left[q_{0}, 1-q_{0}\right]\right) \geqslant 3 \ln 2
$$

which, together with $f_{0} \geqslant 2 \ln 2$ proves the thesis (17) when replaced into Eq. A15.

\section{Derivation of Eq. (22)}

The proof proceeds as the previous ones: we evaluate the left-hand-side of Eq. (22) on the state $\left|\Psi_{\text {sep }}\right\rangle$ of Eq.(8) and we look for its minimum value. Each of the operators (20) has three orthogonal eigenspaces (relative to the non degenerate eigenvalues \pm 2 and the two time degenerate eigenvalue 0), so that its entropic uncertainties (2) has three contributions, i.e. 


$$
\begin{gathered}
H\left(\mathbb{S}_{1},\left|\Psi_{\text {sep }}\right\rangle\right)=\mathcal{F}\left(|\cos \alpha \sin \beta|^{2}+|\sin \alpha \cos \beta|^{2}\right)+\mathcal{F}\left(|\cos \alpha \cos \beta|^{2}\right)+\mathcal{F}\left(|\sin \alpha \sin \beta|^{2}\right) \\
H\left(\mathbb{S}_{2},\left|\Psi_{\text {sep }}\right\rangle\right)=\mathcal{F}[(1-\sin \delta \sin \gamma \sin (2 \alpha) \sin (2 \beta)) / 2]+\mathcal{F}\{[(1-\sin \delta \sin (2 \alpha))(1-\sin \gamma \sin (2 \beta)] / 4\} \\
+\mathcal{F}\{[(1+\sin \delta \sin (2 \alpha))(1+\sin \gamma \sin (2 \beta)] / 4\} \\
H\left(\mathbb{S}_{3},\left|\Psi_{\text {sep }}\right\rangle\right)=\mathcal{F}[(1-\cos \delta \cos \gamma \sin (2 \alpha) \sin (2 \beta)) / 2]+\mathcal{F}\{[(1-\cos \delta \sin (2 \alpha))(1-\cos \gamma \sin (2 \beta)] / 4\} \\
+\mathcal{F}\{[(1+\cos \delta \sin (2 \alpha))(1+\cos \gamma \sin (2 \beta)] / 4\},
\end{gathered}
$$

with $\mathcal{F}(x) \equiv-x \ln x$. By summing these terms we obtain the total entropic uncertainty of the operators $\hat{S}_{j}$ on the state $\left|\Psi_{\text {sep }}\right\rangle$. An analytical study of the resulting expression is very demanding: however a simple numerical analysis shows that the minimum of this function is $3 \ln 2$ (see Fig. 21). Notice that the inequality (22) becomes an identity by choosing (for instance) $\alpha=0$ and $\beta=\pi / 2$, i.e. $\left|\Psi_{\text {sep }}\right\rangle=|01\rangle$.

\section{APPENDIX B: PROPERTIES OF THE MAXIMALLY ENTANGLED STATES}

In this section we derive some useful relations for the maximally entangled states introduced in Sec. III

Superposition with separable states:- First of all we show that given a maximally entangled state $\left|\Psi_{b e l l}\right\rangle$ and a generic separable state $\rho_{\text {sep }}$ of $\mathcal{S}$ we have

$$
\left\langle\Psi_{\text {bell }}\left|\rho_{\text {sep }}\right| \Psi_{\text {bell }}\right\rangle \leqslant 1 / d,
$$

where $d$ is dimension of the subsystems $\mathcal{A}$ and $\mathcal{B}$. Since $\rho_{\text {sep }}$ can be expressed as a convex convolution of pure separable states, to prove Eq. (B1) it is sufficient to show that it holds for any $\left|\Psi_{\text {sep }}\right\rangle=|\psi\rangle_{a} \otimes|\phi\rangle_{b}$. Consider an orthonormal basis $\left\{\left|\psi_{i}\right\rangle_{a}\right\}$ of $\mathcal{A}$ and an orthonormal basis $\left\{\left|\phi_{l}\right\rangle_{b}\right\}$ of $\mathcal{B}$ (here $i, l=1, \cdots, d$ ) and construct the orthonormal basis of $\mathcal{S}$ made of the separable pure states $\left|\psi_{i}\right\rangle_{a} \otimes\left|\phi_{l}\right\rangle_{b} \equiv\left|\psi_{i}, \phi_{l}\right\rangle$. Expanding $\left|\Psi_{\text {bell }}\right\rangle$ in this basis and using the property (31) one can verify that the probabilities $Q_{i l} \equiv\left|\left\langle\Psi_{\text {bell }} \mid \psi_{i}, \phi_{i}\right\rangle\right|^{2}$ satisfy the relations

$$
\sum_{i^{\prime}=1}^{d} Q_{i^{\prime} l}=\sum_{l^{\prime}=1}^{d} Q_{i l^{\prime}}=1 / d,
$$

for all $i$ and $l$. Since $Q_{i l}$ are positive quantities, Eq. (B2) implies that each of them cannot be greater than $1 / d$. This proves the thesis (B1) since the vectors $\left|\psi_{i}\right\rangle_{a}$ and $\left|\phi_{l}\right\rangle_{b}$ can be chosen arbitrarily.

Basis of maximally entangled states:- An orthonormal basis of maximally entangled state can be constructed from the separable basis $\left\{\left|\psi_{i}, \phi_{l}\right\rangle\right\}$ of $\mathcal{S}$ introduced in the previous section. For instance, divide the elements of the separable basis into $d$ orthogonal subsets, the first containing the states $\left|\psi_{i}, \phi_{i \oplus 1}\right\rangle$ with $i=1, \cdots d$ (here $\oplus$ is sum modulus $d$ ), the second containing the states $\left|\psi_{i}, \phi_{i \oplus 2}\right\rangle$ with $i=1, \cdots d$, etc. Now from each of these subsets we can extract $d$ orthogonal maximally entangled state by applying a quantum Fourier transform [1] to the subset elements: the orthogonality between the subsets guarantees that at the hand we have generated $d \times d$ orthogonal maximally entangled states, i.e. a basis of $\mathcal{S}$.
[1] M.I Nielsen and I.L. Chuang, Quantum Computation and Quantum Information (Cambridge University Press, Cambridge, 2000)

[2] A. Peres, Phys. Rev. Lett. 77, 1413 (1996); P. Horodecki, Phys. Lett. A 232, 333 (1997).

[3] M. Horodecki, P. Horodecki, and R. Horodecki, Phys. Lett. A 223, 8 (1996); M. Lewenstein, B. Kraus, J. I. Cirac, and P. Horodecki, Phys. Rev. A 62, 052310 (2000).

[4] P. Horodecki and A. Ekert, Phys. Rev. Lett. 89, 127902 (2002).

[5] R. Simon, Phys. Rev. Lett. 84, 2726 (2000).

[6] L. M. Duan, G. Giedke, J. I. Cirac and P. Zoller, Phys. Rev. Lett. 84, 2722 (2000).

[7] M. D. Reid, e-print quant-ph/0112038 M. D. Reid and P. D. Drummond, Phys. Rev. Lett. 60, 2731 (1988); P. D. Drummond and M. D. Reid, Phys. Rev. A 41, 3930 (1990); M. D. Reid, Phys. Rev. A 40, 913, (1989).

[8] V. Giovannetti, S. Mancini, D. Vitali, and P. Tombesi
Phys. Rev. A 67, 022320 (2003).

[9] N. Korolkova, and R. Loudon, eprint quant-ph/ 0303135 N. Korolkova, et al. Phys. Rev. A 65, 052306 (2002); Korolkova, et al. in Quantum Information Theory with Continuous Variables, edited by S. L. Braunstein and A. K. Pati, (Kluwer Academic, Dordrecht, 2001).

[10] H. F. Hofmann and S. Takeuchi, Phys. Rev. A 68, 032103 (2003).

[11] N.J Cerf and C. Adami, Phys. Rev. A 553371 (1997).

[12] N.J. Cerf and C. Adami, eprint quant-ph/9605002

[13] D. Deutsch, Phys. Rev. Lett. 50, 631, (1983).

[14] V. Majerník and L. Richterek, Eur. J. Phys. 18, 79, (1997).

[15] H. Maassen and J.B.M. Uffink, Phys. Rev. Lett. 60, 1103 (1988).

[16] J. Sánchez, Phys. Lett. A 173, 233 (1993); ibid. 201, 125 (1995); ibid. 244, 189 (1998).

[17] M. Krishna and K.R. Parthasarathy, Sankhya A64, 842 
(2002), eprint quant-ph/0110025

[18] The standard expression $H(\hat{X}, \rho)$ for the entropic uncertainty of the operator $\hat{X}$ is given by the Shannon entropy of the projection probabilities of $\rho$ over a particular basis of eigenstates of $\hat{X}$ [13, 14, 15, 16]. In other words, consider an orthonormal basis of eigenvectors $\left\{\left|x_{k, m}\right\rangle\right\}$ of the operator $\hat{X}$, such that $\hat{X}\left|x_{k, m}\right\rangle=x_{k}\left|x_{k, m}\right\rangle$, with $x_{k}$ eigenvalue of degeneracy $M_{k}$. One defines

$$
H(\hat{X}, \rho) \equiv-\sum_{k=1}^{K} \sum_{m=1}^{M_{k}} P_{k, m} \ln P_{k, m},
$$

where $P_{k, m} \equiv\left\langle x_{k, m}|\rho| x_{k, m}\right\rangle$ is the probability associated with the $k, m$ th basis element. The quantity (B3) coincides with $H(\mathbb{X}, \rho)$ of Eq. (2) only if $\hat{X}$ is non degenerate (i.e. if $M_{k}=1$ for all $k$ ), otherwise one can show that $H(\hat{X}, \rho) \geqslant H(\mathbb{X}, \rho)$. Moreover for degenerate observables, the definition of $H(\hat{X}, \rho)$ depends on the particular choice of the set $\left\{\left|x_{k, m}\right\rangle\right\}$, while $H(\mathbb{X}, \rho)$ clearly does not.

[19] T. M. Cover, and J. A. Thomas, Elements of Information Theory (Wiley, New York, 1991).

[20] The condition (7) does not depend on the choice of the basis $\{|0\rangle,|1\rangle\}$ : we can thus generalize this relation to the case where $\hat{X} \equiv \sigma_{a}\left(\hat{n}_{a}\right) \otimes \sigma_{b}\left(\hat{n}_{b}\right), \hat{Y} \equiv \sigma_{a}\left(\hat{n}_{a}^{\perp}\right) \otimes \sigma_{b}\left(\hat{n}_{b}^{\perp}\right)$, $\sigma_{s}\left(\hat{n}_{s}\right)$ being the Pauli operator of the $s$ qubit in the $\hat{n}_{s}$ direction, and $\hat{n}_{s}^{\perp}$ a unit vector orthogonal to $\hat{n}_{s}$.

[21] R. F. Werner, Phys. Rev. A 40, 4277 (1989); Y.-S. Zhang et al., Phys. Rev. A 66, 062315 (2002).

[22] To show that the separability criterion given in 8] recognizes the state $w_{p}$ of Eq. (23) as entangled only if $p>1 / 2$, define the operators

$$
\begin{aligned}
& \hat{U}=\sigma_{a}^{(1)} \otimes \mathbb{1}_{b}-\mathbb{1}_{a} \otimes \sigma_{b}^{(1)} \\
& \hat{V}=\sigma_{a}^{(2)} \otimes \mathbb{1}_{b}-\mathbb{1}_{a} \otimes \sigma_{b}^{(2)} .
\end{aligned}
$$

According to Eq. (22) of [8] any separable state $\rho_{\text {sep }}$ must satisfies the inequality

$$
\left\langle\Delta^{2} \hat{U}\right\rangle\left\langle\Delta^{2} \hat{V}\right\rangle \geqslant 4\left|\left\langle\sigma_{a}^{(3)} \otimes \sigma_{b}^{(3)}\right\rangle\right|^{2},
$$

where $\langle\cdots\rangle \equiv \operatorname{Tr}\left[\cdots \rho_{\text {sep }}\right]$ and $\Delta^{2} \hat{U} \equiv \hat{U}^{2}-\langle\hat{U}\rangle^{2}$. When applied to the state $w_{p}$ this inequality gives $(1-p)^{2} \geqslant p^{2}$ which is verified for all $p \leqslant 1 / 2$.

[23] P. W. Shor, eprint quant-ph/0305035 (2003); A. S. Holevo and M. E. Shirokov, eprint quant-ph/0306196 (2003); V. Giovannetti, S. Guha, S. Lloyd, L. Maccone, J. H. Shapiro, Phys. Rev. A accepted for publication, eprint quant-ph/0404005 